\title{
Calcite: Kinetics of Two-step Thermal Decomposition
}

\author{
Di WANG ${ }^{1, a}$, Guang-Lei TAN ${ }^{2, b}$ Zhen $Z \mathrm{HAO}^{3, \mathrm{c}}$ and Qi WANG ${ }^{1, \mathrm{~d}, *}$ \\ ${ }^{1}$ Key Laboratory of Chemical Metallurgy Engineering, Liaoning Province, University of Science and \\ Technology Liaoning, Anshan, Liaoning, China \\ ${ }^{2}$ Department of chemical engineering, Yingkou institute of technology, Yingkou, Liaoning, China \\ ${ }^{3}$ School of Chemistry and Life Science, Anshan Normal University, Anshan, Liaoning, China \\ awangdinow@163.com, b229293343@qq.com, czhaozhenlunwen@yeah.net, dwangqi8822@sina.c \\ om \\ ${ }^{*}$ Corresponding author
}

Keywords: Calcite, Thermal decomposition, Kinetics, Reaction mechanism, Activation energy.

\begin{abstract}
Thermal decomposition of calcium carbonate has been studied by thermogravimetric analysis (TGA). The complementary application of the isoconversional method and model-fitting method has been used for deriving the kinetic triplet (activation energy $[E]$, frequency factor $[A]$, and model) from the TGA experiment. The conversion dependence of activation energy obtained from the isoconversional method suggested that the decomposition process was divided into two steps. For each step, it was based on using the isoconversional method to select the reaction model, and then with using the model-fitting method the frequency factor can be obtained. By determining two sets of the kinetic triplets, it can be derived that the steps of $\mathrm{CaCO}_{3}$ decomposition change from nuclei growth to phase boundary reaction.
\end{abstract}

\section{Introduction}

The thermal decomposition of calcium carbonate is an important solid-state reaction for industrial production. The kinetics of this reaction has been extensively investigated [1-6]. This thermal decomposition process is known $[7,8]$ to be a tangled interplay of various chemical and physical phenomena. Because the ratio of the contributions of these involved elementary steps to the overall reaction rate is determined by the experimental conditions, the reported effective activation energy shows a dependence on experimental parameters [9]. Maciejewski [10] showed that the majority of reported values of the effective activation energy fit into the interval $120-280 \mathrm{~kJ} \cdot \mathrm{mol}^{-1}$. Even for the same set of TGA experiments, different kinetic information is derived by different kinetic analyzing methods [4].

Under nonisothermal conditions, model-fitting and isoconversional methods are two alternative approaches for analyzing solid-state kinetic data. The constant activation energy values obtained by mode-fitting methods are in fact averages for the wide extents of reaction conversion, that are rather insensitive to the changes in the mechanism and kinetics with the extent of conversion [11,12]. The dependence of the activation energy $\left(E_{\alpha}\right)$ on the extent of conversion $(\alpha)$ represented by the isoconversional method is able to reveal the multi-step nature of solid-state reaction, which plays an important role in solid-state kinetic analysis [13]. The Coats-Redfern method [14] is reported to be one of the most frequently used model-fitting methods to process nonisothermal data [15]. The isoconversional method can be divided into two groups, linear [16] and non-linear [17,18], and the calculation accuracy of the latter is higher [17].

In this work, the advanced non-linear isoconversional method [18] has been employed to investigate the multistep kinetics of the non-isothermal decomposition of calcium carbonate. The main purpose of this work is to detect the different reaction steps probably involved. Based on the complementary use of isoconversional and model-fitting methods for each stage, all kinetic parameters have been derived, and a full kinetic description for the nonisothermal decomposition of $\mathrm{CaCO}_{3}$ has been realized. 


\section{Theory and Methods}

Kinetic analysis of solid-state decompositions under nonisothermal conditions are usually based on a single step equation

$$
\frac{d \alpha}{d T}=\frac{A}{\beta} \exp \left(-\frac{E}{R T}\right) f(\alpha)
$$

where $T$ is the temperature, $\beta$ is the heating rate, $\alpha$ is the extent of conversion, $f(\alpha)$ is a function that represents the reaction model, $A$ is the frequency factor, $E$ is the activation energy, $R$ is the gas constant.

Eq. 1 can be rearranged and integrated to obtain

$$
g(\alpha)=\frac{A}{\beta} \int_{0}^{T} \exp \left(-\frac{E}{R T}\right) d T=\frac{A}{\beta} I(E, T)
$$

which is the integrated form of the reaction model that is commonly used to describe the kinetics of reaction in solid. Several examples of reaction models are listed in Table 1, the exponential integral $I(E, T)$ has no analytic solution [19], but has many approximations, the 3th degree Senum-Yang approximation [20] is adopted here, as

$$
I(E, T)=T \cdot \exp (-u) \cdot\left(\frac{u^{2}+10 u+18}{u^{3}+12 u^{2}+36 u+24}\right)
$$

where $u=E / R T$.

Table 1. Various mechanistic functions with integral form examined in this work

\begin{tabular}{lll}
\hline symble & $g(\alpha)$ & Rate-controlling process \\
\hline Am & $(-\ln (1-\alpha))^{1 / m}$ & Nucleation and nuclei growth $(m=1,4 / 3,2$, and 3) \\
Rn & $1-(1-\alpha)^{1 / n}$ & Phase boundary reaction $(n=1,4 / 3,2$, and 3) \\
D1 & $\alpha^{2}$ & \\
D2 & {$[(1-\alpha) \ln (1-\alpha)]+\alpha$} & Diffusion of gaseous product \\
D3 & {$\left[1-(1-\alpha)^{1 / 3}\right]^{2}$} & \\
\hline
\end{tabular}

Methods for analyzing solid-state kinetic data can be grouped into isoconversional and model-fitting methods. Isoconversional methods calculate $E_{\alpha}$ values at the extent of conversion without modelistic assumptions, by analyzing the $E_{\alpha}$ dependence the complexity of solid-state reaction can be revealed. The advanced nonlinear isoconversional method [18] is a nonisothermal method that uses an accurate, nonlinear, Senum-Yang approximation of exponential integral, which leads to,

$$
\Omega=\left|\sum_{i=1}^{n} \sum_{j \neq i}^{n} \frac{\beta_{j} J\left(E_{\alpha}, T_{\alpha, i}\right)}{\beta_{i} J\left(E_{\alpha}, T_{\alpha, j}\right)}\right|
$$

where $J\left(E_{\alpha}, T_{\alpha}\right) \equiv \int_{T_{\alpha-\Delta \alpha}}^{T_{\alpha}} \exp \left(-\frac{E_{\alpha}}{R T}\right) d T=I\left(E_{\alpha}, T_{\alpha}\right)-I\left(E_{\alpha}, T_{\alpha-\Delta \alpha}\right)$

At an extent of conversion the activation energy is the value that minimizes $\Omega$ in Eq. 4 . 
The Coats-Redfern method [14] utilizes the asymptotic series expansion in approximating exponential integral, producing the following equation,

$$
\ln \frac{g(\alpha)}{T^{2}}=\ln \left(\frac{A R}{\beta E}\left(1-\left(\frac{2 R T_{\exp }}{E}\right)\right)\right)-\frac{E}{R T}
$$

where $T_{\exp }$ is the mean experimental temperature. Plotting the left-hand side (which includes the model $g(\alpha)$ ) of Eq. 6 versus 1/T gives Arrhenius parameters ( $E$, and $A$ ) from the slop and intercept, respectively.

Combining the power of the isoconversional method and model-fitting method, the complementary application of these two methods uses isoconversional methods to obtain $E_{\alpha}$ values which are compared to values obtained by model-fitting methods. The most accurate model is seen as the one that provides the calculation results closet to that from the isoconversional analysis.

\section{Experimental Section}

In this work, a sample of $\mathrm{CaCO}_{3}$ powder was supplied by Sigma-Aldrich, Inc. (Lot Number MKBL0094V), average particle size of sample was $40 \mu \mathrm{m}$, and the sample weight was $2.0 \pm 0.1 \mathrm{mg}$. The thermogravimetric analysis (TGA) experiments were carried out using a Setaram SETSYS Evolution instrument with the weighing sensitivity of $0.1 \mu \mathrm{g}$. Samples were heated in an open $5 \mathrm{~mm}$ diameter platinum crucible under flowing high purity (99.99\%) nitrogen (30 $\mathrm{ml} \cdot \mathrm{min}^{-1}$ ) carrier gas. An empty platinum crucible was used as reference. The experimental temperature increased from room temperature to $1000^{\circ} \mathrm{C}$ with five constant heating rates of $2.5,5,7.5,10$, and $15 \mathrm{~K} \cdot \mathrm{min}^{-1}$ respectively.

Kinetic analysis of the experimental data was conducted by isoconversional and model-fitting methods. The advanced nonlinear isoconversional method and Coats-Redfern model-fitting method were used to analyze the nonisothermal data. Model selection was done by the comparing of the activation energy calculation results from the two methods, the model for which the values matched that from the isoconversional analysis was selected as the accurate model. TGA curves were reconstructed according to Eq. 2. The accuracy of the kinetic triplet selected by the isoconversional method for each heating rate was verified by the good correlation between the reconstructed and actual experimental curves.

\section{Results and Discussions}

Fig. 1 shows the TGA curves of the $\mathrm{CaCO}_{3}$ decomposition under different heating rates. As shown in Fig. 2a, the $E_{\alpha}$ dependence calculated by the advanced nonlinear isoconversional method [18] exhibits a marked increase from $\sim 206$ to $\sim 221 \mathrm{~kJ} \cdot \mathrm{mol}^{-1}$ at the initial stage then keeps constant by the completion of decomposition. Both the maximum and minimum $E_{\alpha}$ values here reported are in agreement with those most commonly reported in the literature [10].

Here the $E_{\alpha}$ dependence is smoothed and for taking a derivative, as shown in Fig. 2b, the result of derivation is approximately zero from $\alpha=0.16$. The $E_{\alpha}$ dependence can be divided into two parts by $\alpha=0.16$, i.e., the initial increasing $E_{\alpha}$ dependence in the range of $0.02 \leq \alpha<0.16$ (stage I ), and the flat $E_{\alpha}$ dependence in the range of $0.16 \leq \alpha \leq 0.9$ (stage $\mathbb{I}$ ). 


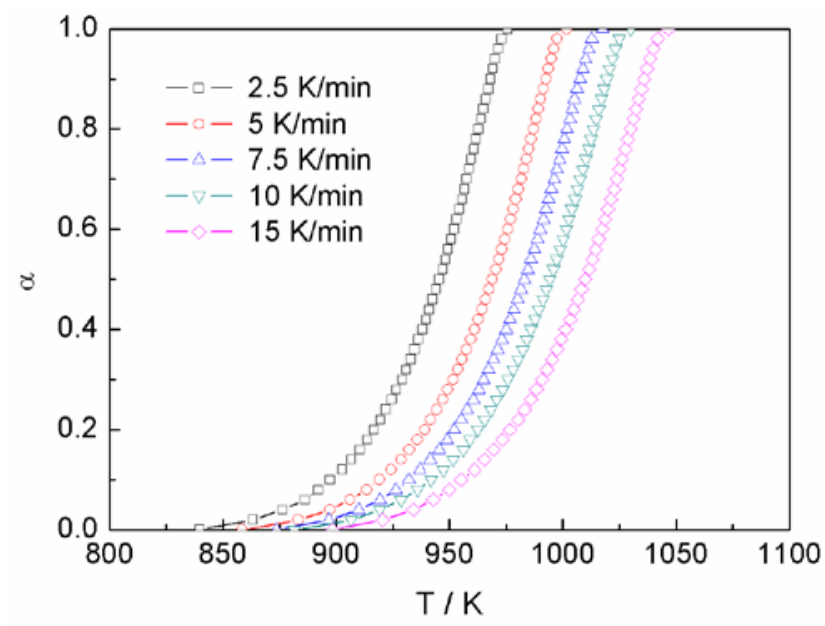

Fig. 1. TGA curves for the thermal decomposition of $\mathrm{CaCO}_{3}$ at different heating rates.
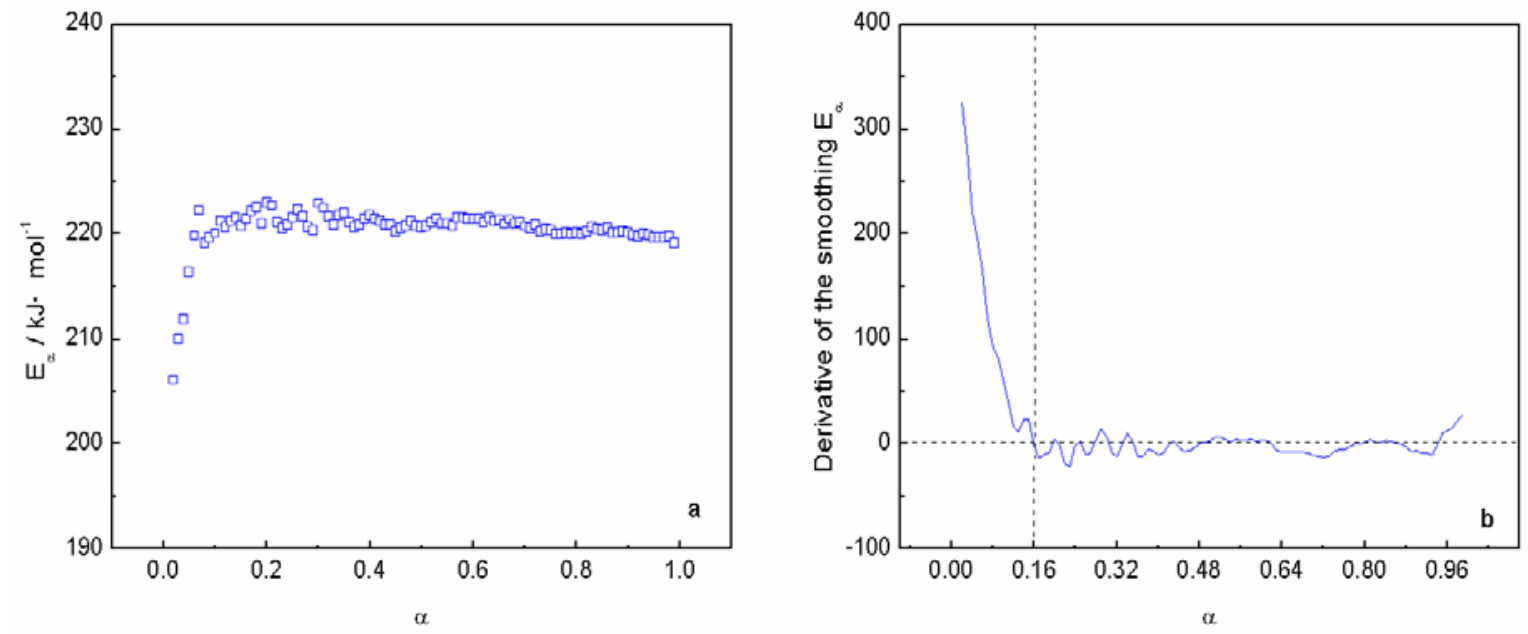

Fig. 2. (a) $E_{\alpha}$ dependence for the $\mathrm{CaCO}_{3}$ decomposition determined by the advanced nonlinear isoconversional method ( $\square$ ). (b) Derivation result of the $E_{\alpha}$ dependence in this work.

For the models listed in Table 1, the Arrhenius parameters determined from the nonisothermal TGA data of stage I and II using Coats-Redfern model-fitting method are listed in Table 2 and 3. For stage $\mathrm{I}$ and $\mathbb{I}$, plots containing activation energies calculated by model-fitting along with that from the advanced nonlinear isoconversional method are shown in Fig. 3.

Table 2. Fitted kinetic parameters for $\mathrm{CaCO}_{3}$ nonisothermal decomposition of stage I $(0.02 \leq \alpha<0.16)$, using the Coates-Redfern method ${ }^{\mathrm{a}}$

\begin{tabular}{cccc}
\hline model & $\ln A\left(\mathrm{~s}^{-1}\right)$ & $E\left(\mathrm{~kJ} \cdot \mathrm{mol}^{-1}\right)$ & $r^{2}$ \\
\hline A1 & $29.50 \pm 0.70$ & $286 \pm 6$ & -0.9983 \\
$\mathrm{~A} 4 / 3$ & $20.58 \pm 1.18$ & $215 \pm 10$ & -0.9983 \\
A2 & $10.77 \pm 1.08$ & $138 \pm 7$ & -0.9982 \\
A3 & $4.09 \pm 1.01$ & $87 \pm 5$ & -0.9979 \\
R1 & $28.64 \pm 1.36$ & $282 \pm 13$ & -0.9977 \\
R4/3 & $28.74 \pm 1.36$ & $284 \pm 13$ & -0.9979 \\
\hline
\end{tabular}




\begin{tabular}{llll}
\hline R2 & $28.72 \pm 1.37$ & $287 \pm 13$ & -0.9980 \\
R3 & $28.56 \pm 1.38$ & $289 \pm 13$ & -0.9982 \\
D1 & $65.10 \pm 2.15$ & $578 \pm 25$ & -0.9978 \\
D2 & $65.40 \pm 2.18$ & $585 \pm 25$ & -0.9981 \\
D3 & $64.92 \pm 2.20$ & $593 \pm 25$ & -0.9983
\end{tabular}

${ }^{\mathrm{a}}$ Results of $\ln A$ and $E$ are value ranges from five heating rates, relusts of $r^{2}$ are averaged from five curves by taking the arithmetic mean.

Table 3. Fitted kinetic parameters for $\mathrm{CaCO}_{3}$ nonisothermal decomposition of stage II $(0.16 \leq \alpha \leq 0.9)$, using the Coates-Redfern method ${ }^{\mathrm{a}}$

\begin{tabular}{cccc}
\hline model & $\ln A\left(\mathrm{~s}^{-1}\right)$ & $E\left(\mathrm{~kJ} \cdot \mathrm{mol}^{-1}\right)$ & $r^{2}$ \\
\hline A1 & 31.44 & 303 & -0.9951 \\
A4/3 & 21.45 & 223 & -0.9949 \\
A2 & 11.34 & 143 & -0.9946 \\
A3 & 4.48 & 90 & -0.9940 \\
R1 & 18.35 & 202 & -0.9990 \\
R4/3 & 20.90 & 224 & -0.9999 \\
R2 & 23.62 & 248 & -0.9995 \\
R3 & 25.46 & 265 & -0.9986 \\
D1 & 45.15 & 421 & -0.9991 \\
D2 & 51.62 & 476 & -0.9999 \\
D3 & 59.11 & 546 & -0.9986
\end{tabular}

${ }^{\mathrm{a}}$ Results averaged from five curves of different heating rates by taking the geometrical mean of $\ln A$ and $E$ and arithmetic mean of $r^{2}$.
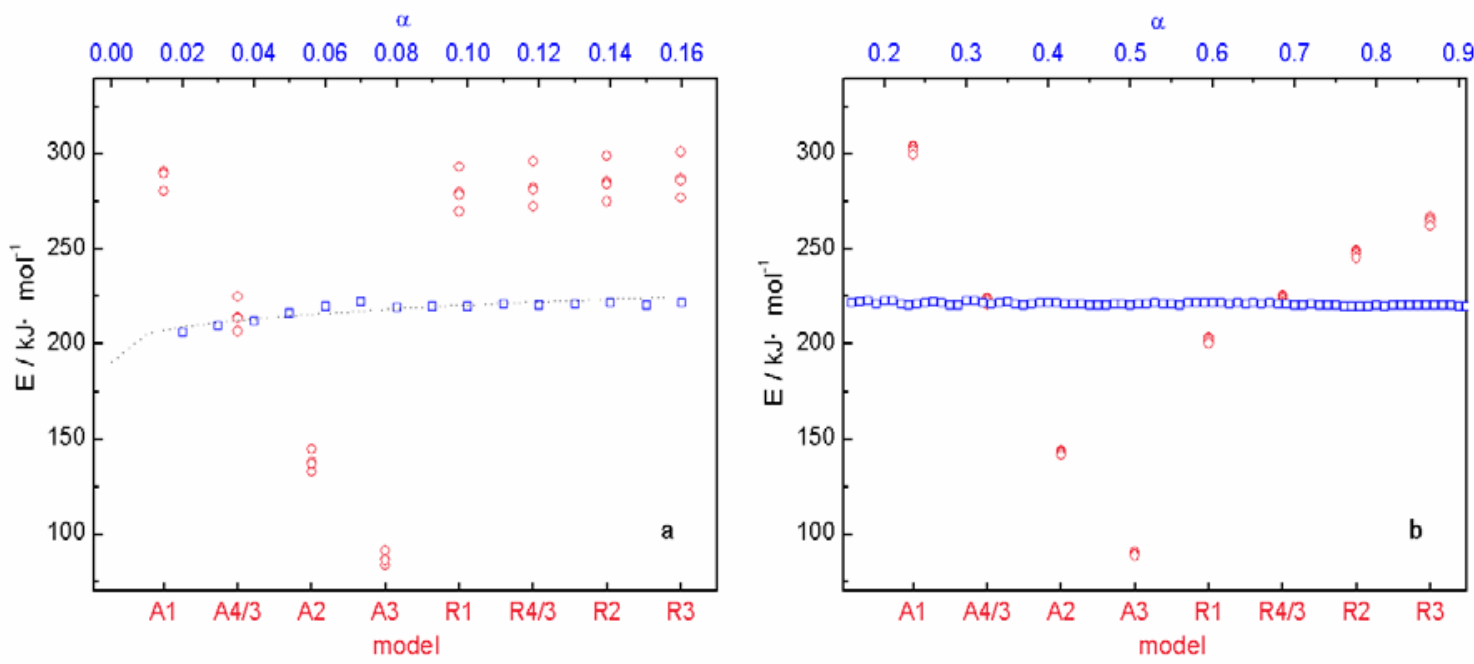

Fig. 3. Activation energies calculated by Coats-Redfern model-fitting method ( () ) and the advanced nonlinear isoconversional method ( $\square$ ). (a: stage I ; b: stage II). 
In stage I , as shown in Fig. 3a, the value range of model-fitting results with model A4/3 is about the same as which of the isoconversional computing result, therefore the nucleation and nuclei growth A4/3 model is chosen as the reaction model for this stage.

As known the process of nuclei growth occurs as multiple steps [21]. Tan et al. [22] has deduced the variable activation energy expression for nuclei growth as

$$
E_{\alpha}^{v a}=E_{D}+C \alpha^{n_{c}}
$$

where $E_{\alpha}{ }^{v a}$ is the variable activation energy for nuclei growth, $E_{D}$ is the activation energy of diffusion of nuclei particle, $C$ is constant with intrinsic property, and $n_{\mathrm{c}}$ is $1 / 3$.

Assuming that the deduced model A4/3 manifests nuclei growth is the rate-controlling step at stage $\mathrm{I}$, the increasing $E_{\alpha}$ dependence may be expressed as the form of Eq. 7 approximately. Where $E_{D}$ is about $191 \mathrm{~kJ} \cdot \mathrm{mol}^{-1}$, derived by extrapolating the determined $E_{\alpha}$ dependence to $\alpha \rightarrow 0, C$ and $n_{c}$ are about $59 \mathrm{~kJ} \cdot \mathrm{mol}^{-1}$ and 0.3 , obtained by the slope and intercept of the linearity of the plot $\ln \left(E_{\alpha}-191\right)$ against $\ln \alpha$. It leads to

$$
E_{\alpha}(\alpha)=191+59 \times \alpha^{0.3}
$$

By an artificial isokinetic relationship [13,23] from the kinetic parameters for model A4/3 with different heating rates, which is listed in Table 4, the dependence of $\ln A_{\alpha}$ on $\alpha$ is obtained:

$$
\ln A_{\alpha}(\alpha)=17.46+8.97 \alpha^{0.3}
$$

Table 4. Kinetic parameters for the model A4/3 with different heating rates

\begin{tabular}{lll}
\hline$\beta\left(\mathrm{K} \cdot \mathrm{min}^{-1}\right)$ & $\ln A\left(\mathrm{~s}^{-1}\right)$ & $E\left(\mathrm{~kJ} \cdot \mathrm{mol}^{-1}\right)$ \\
\hline 2.5 & 19.42 & 206.75 \\
5 & 19.41 & 206.65 \\
7.5 & 20.45 & 214.42 \\
10 & 20.29 & 213.43 \\
15 & 21.76 & 224.82 \\
\hline $\ln A=0.13 E-7.37$ & $r^{2}=0.9999$ & \\
\hline
\end{tabular}

In stage $\mathbb{I}$, Fig. 3b shows that, the isoconversional curve intersects values for both the A4/3 and R4/3 models, and Table 3 shows that the $r^{2}$ value of R4/3 model is higher than A4/3 model. Therefore, the phase boundary reaction R4/3 model is selected as the reaction model for this stage.

Table 5. Two kinetic triplets derived from the $\mathrm{CaCO}_{3}$ decomposition process

\begin{tabular}{lll}
\hline & stage I & stage II \\
& $(0.02 \leq \alpha<0.16)$ & $(0.16 \leq \alpha \leq 0.9)$ \\
\hline$E_{\alpha}\left(\mathrm{kJ} \cdot \mathrm{mol}^{-1}\right)$ & $E_{\alpha}=191+59 \times \alpha^{0.3}$ & 224 \\
$\ln A\left(\mathrm{~s}^{-1}\right)$ & $\ln A_{\alpha}=17.46+8.97 \alpha^{0.3}$ & 20.90 \\
$\mathrm{~g}(\alpha)$ & $(-\ln (1-\alpha))^{3 / 4}$ & $1-(1-\alpha)^{3 / 4}$ \\
\hline
\end{tabular}


Kinetic triplets of stage I and $I I$ are listed in Table 5, according to Eq.2, the accuracy of which has been verified by the reconstruction results for each heating rate. It can be seen from Fig. 4, the reconstructed results are in good agreement with the experimental data. A two-step mechanism for $\mathrm{CaCO}_{3}$ decomposition has been derived in this work, i.e., the steps of the decomposition process change from nuclei growth to phase boundary reaction.

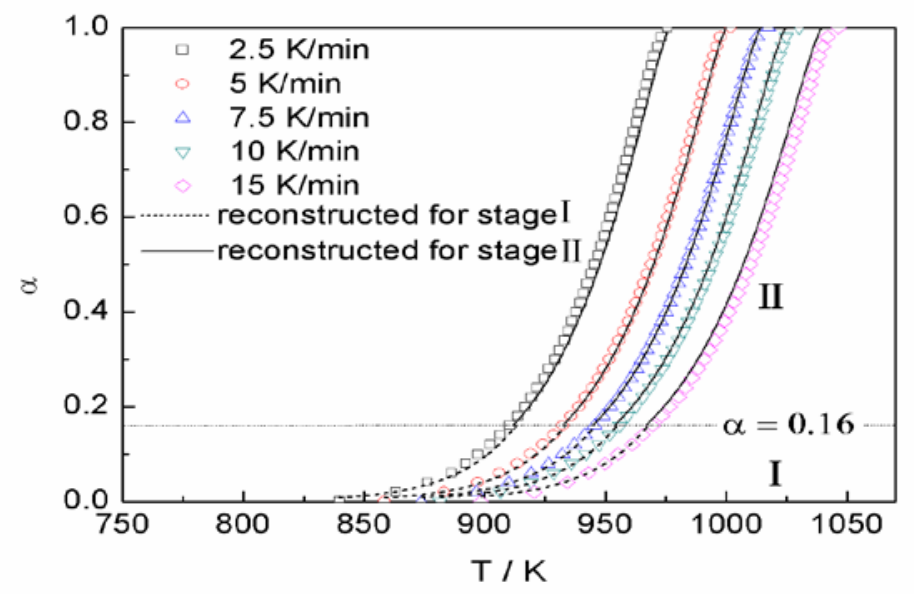

Fig. 4. Reconstructed TGA curves. Experimental data are represented as symbols, solid lines represent the $\alpha$-T curves of stage I reconstructed for model A4/3, dot lines represent the $\alpha$-T curves of stage II reconstructed for model R4/3.

\section{Conclusions}

For the nonisothermal $\mathrm{CaCO}_{3}$ decomposition, the multi-step reaction mechanism can be reflected in the conversion dependence of activation energy. The obtained dependence with an increasing shape at the initial and then turn to flat may suggest that two steps occur in the decomposition process successively. Based on the isoconversional result the reaction model for each step has been selected, thereafter each frequency factor can be obtained by the model-fitting method. Based on the complementary application of the isoconversional method and model-fitting method, two sets of kinetic triplets have been derived for the kinetic description of the decomposition reaction of calcite. The two-step kinetic analysis result can be explained by considering that a complete nucleation on the interface, reaction occurs at a low extent of conversion, at which the inward advance of the interface is started.

\section{Acknowledgement}

This research was financially supported by the Educational Department of Liaoning Province (No. L2015552).

\section{References}

[1] Rao, T.R., Kinetics of calcium carbonate decomposition, Chem. Eng. Technol. 19 (1996) 373-377.

[2] Liu, R., et al., Kinetics and mechanism of decomposition of nano-sized Calcium Carbonate under Non-isothermal Condition, Chinese J. Chem. Eng. 11 (2003) 302-306. 
[3] Dollimore, D., P. Tong, and K.S. Alexander, The kinetic interpretation of the decomposition of calcium carbonate by use of relationships other than the Arrhenius equation, Thermochim. Acta 282-283 (1996) 13-27.

[4] Brown, M.E., et al., Computational aspects of kinetic analysis: Part A: The ICTAC kinetics project-data, methods and results, Thermochim. Acta 355 (2000) 125-143.

[5] Maitra, S., et al., Non-isothermal decomposition kinetics of alkaline earth metal carbonates, J. Am. Ceram. Soc. 90 (2007) 1299-1303.

[6] Sanders, J.P. and P.K. Gallagher, Kinetic analyses using simultaneous TG/DSC measurements: Part I: decomposition of calcium carbonate in argon, Thermochim. Acta 388 (2002) 115-128.

[7] Vyazovkin, S., Handbook of thermal analysis and calorimetry, in: E.B. Michael, K.G. Patrick, (Eds.), Elsevier Science B.V., 2008, pp. 503-538.

[8] Rodriguez-Navarro, C., et al., Thermal decomposition of calcite: Mechanisms of formation and textural evolution of CaO nanocrystals, Am. Min. 94 (2009) 578-593.

[9] Maciejewski, M., Computational aspects of kinetic analysis.: Part B: The ICTAC Kinetics Project - the decomposition kinetics of calcium carbonate revisited, or some tips on survival in the kinetic minefield, Thermochim. Acta 355 (2000) 145-154.

[10]Maciejewski, M. and A. Reller, How (UN)reliable are kinetic data of reversible solid-state decomposition processes? Thermochim. Acta 110 (1987) 145-152.

[11] Vyazovkin, S. and C.A. Wight, Kinetics in solids, Annu. Rev. Phys. Chem. 48 (1997) 125-149.

[12] Vyazovkin, S. and C.A. Wight, Isothermal and nonisothermal reaction kinetics in Solids: In Search of Ways toward Consensus, J. Phys. Chem. A 101 (1997) 8279-8284.

[13] Vyazovkin, S., A unified approach to kinetic processing of nonisothermal data, Int. J. Chem. Kinet. 28 (1996) 95-101.

[14] Coats, A.W. and J.P. Redfern, Kinetic parameters from thermogravimetric data, Nature 201 (1964) 68-69.

[15] Carr, N.J. and A.K. Galwey, Decomposition reactions of solids (an experiment in reviewing), Thermochim. Acta 79 (1984) 323-370.

[16] Ozawa, T., Initial kinetic parameters from thermogravimetric rate and conversion data, Bull. Chem. Soc. Jpn. 38 (1965) 1881-1886.

[17]Vyazovkin, S. and D. Dollimore, Linear and nonlinear procedures in isoconversional computations of the activation energy of nonisothermal reactions in solids, J. Chem. Inf. Comput. Sci., 36 (1996) 42-45.

[18] Vyazovkin, S., Modification of the integral isoconversional method to account for variation in the activation energy, J. Comput. Chem. 22 (2001) 178-183.

[19]Flynn, J.H., The 'Temperature Integral' — Its use and abuse, Thermochim. Acta, 300 (1997) 83-92.

[20] Senum, G.I. and R.T. Yang, Rational approximations of the integral of the Arrhenius function, J. Therm. Anal. 11 (1977) 445-447.

[21] Vyazovkin, S., Kinetic concepts of thermally stimulated reactions in solids: A view from a historical perspective, Int. Rev. Phys. Chem. 19 (2000) 45-60.

[22] Tan, G., et al., Concept of variable activation energy and its validity in nonisothermal kinetics, J. Phys. Chem. A, 115 (2011) 5517-5524. 
[23] Vyazovkin, S. and W. Linert, False isokinetic relationships found in the nonisothermal decomposition of solids, Chem. Phys. 193 (1995) 109-118. 